\title{
p38a MAP kinase phosphorylates RCAN1 and regulates its interaction with calcineurin
}

\author{
MA Lei ${ }^{1}$, TANG HaiPing ${ }^{2}$, REN Yan ${ }^{3}$, DENG HaiTeng ${ }^{2}$, WU JiaWei ${ }^{2}$ \& WANG ZhiXin ${ }^{1,2 *}$ \\ ${ }^{1}$ National Laboratory of Biomacromolecules, Institute of Biophysics and Graduate University, Chinese Academy of Sciences, \\ Beijing 100101, China; \\ ${ }^{2}$ School of Life Sciences, Tsinghua University, Beijing 100084, China; \\ ${ }^{3}$ Department of Biochemistry and Molecular Biology, Beijing Normal University, Beijing 100875, China
}

Received May 29, 2012; accepted June 2, 2012

\begin{abstract}
RCAN1, also known as DSCR1, is an endogenous regulator of calcineurin, a serine/threonine protein phosphatase that plays a critical role in many physiological processes. In this report, we demonstrate that p38 $\alpha$ MAP kinase can phosphorylate RCAN1 at multiple sites in vitro and show that phospho-RCAN1 is a good protein substrate for calcineurin. In addition, we found that unphosphorylated RCAN1 noncompetitively inhibits calcineurin protein phosphatase activity and that the phosphorylation of RCAN1 by p38 $\alpha$ MAP kinase decreases the binding affinity of RCAN1 for calcineurin. These findings reveal the molecular mechanism by which p38 $\alpha$ MAP kinase regulates the function of RCAN1/calcineurin through phosphorylation.
\end{abstract}

p38a MAP kinase, RCAN1, calcineurin, phosphorylation

Citation: Ma L, Tang H P, Ren Y, et al. p38 $\alpha$ MAP kinase phosphorylates RCAN1 and regulates its interaction with calcineurin. Sci China Life Sci, 2012, 55: 559-566, doi: 10.1007/s11427-012-4340-9

Calcineurin $(\mathrm{CN})$ is a $\mathrm{Ca}^{2+} /$ calmodulin-dependent serine/ threonine protein phosphatase with roles in myriad physiological functions [1-3], including $\mathrm{T}$ cell activation [4], apoptosis [5], skeletal myocyte differentiation [6], neuronal synaptic plasticity [7], memory formation [8], and cardiac health [9]. The most thoroughly characterized calcineurin substrates are the nuclear factor of activated T cells (NFAT) family transcription factors. Two distinct calcineurin docking motifs from NFAT, the PxIxIT and LxVP binding motifs, have been identified [10-13]. The PxIxIT motif is proposed to be the main calcineurin binding site, and it is present in many other calcineurin substrates and regulatory proteins. NFAT transcription factors are expressed in most immune cell types and play a central role in the regulation of cytokine gene expression. The localization of NFAT is regulated by its phosphorylation status. Calcineurin depho-

*Corresponding author (email: zhixinwang@mail.tsinghua.edu.cn) sphorylates multiple residues within the regulatory domain of NFAT, leading to its nuclear translocation and the activation of target genes including PTGS2 (COX-2) and TNF- $\alpha$ (TNFA) [11,14].

The regulation of calcineurin phosphatase activity by cellular inhibitors may have potential as novel modulators for the treatment of calcineurin-related diseases. Several endogenous proteins can inhibit the catalytic activity of calcineurin. Modulatory calcineurin interacting proteins (also called RCAN proteins) are unique among these proteins in terms of their expression pattern, and they function in a negative feedback loop to regulate calcineurin activity. The RCAN proteins comprise a family of endogenous calcineurin regulators that are conserved from yeast to humans and are essential for normal calcineurin signaling. Previous studies have shown that RCAN1 phosphorylation alters its effect on calcineurin. Human RCAN1 is encoded within the Down syndrome critical region on chromosome 21 . It is 
abundantly expressed in the heart, brain, and muscles, and it is overexpressed in the brains of individuals with Down syndrome and Alzheimer's disease [15,16]. Recently, RCAN1 has been highlighted because of its possible functional link with vertebrate embryonic development and tumor progression [17-19].

In the present study, we demonstrate for the first time that $\mathrm{p} 38 \alpha$ MAP kinase directly phosphorylates RCAN1 at five sites (S93, S108, S112, T124 and T153) in vitro. We conducted kinetic studies on the phospho-RCAN1 protein and demonstrated that it is a good substrate for calcineurin. We further show that the phosphorylation of RCAN1 by p38 $\alpha$ MAP kinase decreases its binding affinity for calcineurin, suggesting that phosphorylation may relieve the inhibition of calcineurin signaling by RCAN1.

\section{Materials and methods}

\subsection{Materials}

Tris, bacterial purine nucleotide phosphorylase, the materials used to generate the purine nucleotide phosphorylase substrate 7-methyl-6-thioguanosine (MESG), $\mathrm{CaCl}_{2}$, and $\mathrm{MnCl}_{2}$ were purchased from Sigma (St. Louis, MO). 3-(Nmorpholino) propanesulfonic acid (MOPS), bovine serum albumin (BSA), dithiothreitol (DTT), and $\mathrm{NaCl}$ were obtained from Amresco (Solon, OH). MESG was synthesized as described previously [20].

1.2 Plasmid construction and the expression and purification of mouse calcineurin $\alpha$, RCAN1, p38 $\alpha$ MAP kinase, calmodulin (CaM) and p-inhibitor-1

Mouse CNA $\alpha$ and CNB1 were cloned into pET-21b vectors (Novagen) with a C-terminal $\mathrm{His}_{6}$-tag and a chloromycetin-resistant pACYCDuet vector, respectively. The two plasmids encoding CNA $\alpha$ and CNB1 were co-transformed into BL21 (DE3) competent cells to express a mouse $\mathrm{CN \alpha}$ heterodimer $(\mathrm{mCN} \alpha)$. cDNA encoding mouse RCAN1 was kindly provided by Dr. Beverly Rothermel (University of Texas, Southwestern Medical Center, Dallas, TX, USA), and cloned into a pGEX6P1 expression vector with a C-terminal $\mathrm{His}_{6}$-tag. RCAN1 mutants were constructed using QuikChange site-directed mutagenesis with the pGEX6P1-RCAN1 plasmid as a template. Mouse CaM was cloned into a modified pGEX4T-2 vector containing an N-terminal GST-tag and a PreScission protease cleavage site. The expression and purification of bisphosphorylated p38 $\alpha$ MAP kinase were described previously [21]. Phosphorylated inhibitor-1 was obtained by co-expressing $\mathrm{His}_{6}{ }^{-}$ tagged human inhibitor-1 (1-171) plasmid with non-tagged mouse PKAc. Each protein was expressed at $25^{\circ} \mathrm{C}$ and initially purified by Ni-NTA (Qiagen) or glutathioneSepharose (GE Healthcare) affinity columns, followed by anion exchange and gel filtration chromatography (Source15Q and Superdex-200, GE Healthcare). The affinity tag was proteolytically removed from the fusion proteins as indicated. Protein stocks for kinetic analysis were supplemented with glycerol at a final concentration of $20 \%(\mathrm{v} / \mathrm{v})$. Protein concentrations were determined based on the theoretical molar extinction coefficients at $280 \mathrm{~nm}$.

\subsection{In vitro phosphorylation of wildtype (WT) and mutant RCAN1}

The C-terminally $\mathrm{His}_{6}$-tagged WT or mutant RCAN1 (40 $\mu \mathrm{mol} \mathrm{\textrm {L } ^ { - 1 }}$ each) were phosphorylated in a kinase buffer (50 mmol L ${ }^{-1}$ MOPS, pH 7.4, $100 \mathrm{mmol} \mathrm{L}^{-1} \mathrm{NaCl}, 0.1 \mathrm{mmol} \mathrm{L}^{-1}$ EDTA, $10 \mathrm{mmol} \mathrm{L}^{-1} \mathrm{MgCl}_{2}$, and $1 \mathrm{mmol} \mathrm{L}^{-1} \mathrm{ATP}$ ) with 2 $\mu \mathrm{mol} \mathrm{L} \mathrm{L}^{-1} \mathrm{p} 38 \alpha \mathrm{MAP}$ kinase at $25^{\circ} \mathrm{C}$ for $30 \mathrm{~min}$. The reaction was terminated at $2,5,10$ or $30 \mathrm{~min}$. The proteins were resolved by SDS-PAGE, and protein phosphorylation was indicated by a band shift.

Recombinant GST-RCAN1 proteins were purified with glutathione-Sepharose beads according to the manufacturer's instructions (Amersham Biosciences). The GST-tagged RCAN1 was phosphorylated by p38 $\alpha$ MAP kinase at $25^{\circ} \mathrm{C}$ for $120 \mathrm{~min}$, and the system was replenished with $1 \mathrm{mmol} \mathrm{L}^{-1}$ ATP every $30 \mathrm{~min}$. Then, the proteins were loaded onto glutathione-Sepharose beads and washed three times with buffer $\left(50 \mathrm{mmol} \mathrm{L}^{-1}\right.$ Tris $\mathrm{pH} 8.0$ and $150 \mathrm{mmol} \mathrm{L}^{-1} \mathrm{NaCl}$ ). The GST tags were digested with PreScission protease overnight at $4^{\circ} \mathrm{C}$. Gel filtration chromatography (Superdex-200, GE Healthcare) was performed to further purify the phosphorylated RCAN1.

\subsection{In-gel digestion for mass spectrometry}

To identify phosphorylation sites on p-RCAN1 WT and S108A/S112A, samples were separated by SDS-PAGE. The gel bands corresponding to the targeted proteins were excised from the gel, reduced with $25 \mathrm{mmol} \mathrm{L}^{-1}$ DTT and alkylated with $55 \mathrm{mmol} \mathrm{L}^{-1}$ iodoacetamide. Then, in-gel digestion was performed with sequencing grade modified trypsin (Promega, Fitchburg, WI) in $50 \mathrm{mmol} \mathrm{L}^{-1}$ ammonium bicarbonate at $37^{\circ} \mathrm{C}$ overnight. The peptides were extracted twice with $1 \%$ trifluoroacetic acid in $50 \%$ acetonitrile aqueous solution for $30 \mathrm{~min}$. The extraction mixtures were then concentrated by SpeedVac.

\subsection{Liquid chromatography-mass spectrometry (LC-MS)/ MS analysis}

For LC-MS/MS analysis, the digestion product was separated by a 65 min gradient elution at a flow rate of $0.250 \mu \mathrm{L}$ $\min ^{-1}$ with the EASY-nLCIITM integrated nano-HPLC system (Proxeon, Denmark), which has a direct interface with the Thermo LTQ-Orbitrap mass spectrometer. The analyti- 
cal column was a homemade fused silica capillary column (75 $\mu \mathrm{m}$ ID, 150 mm length; Upchurch, Oak Harbor, WA) packed with C-18 resin (300 $\AA, 5 \mu \mathrm{m}$, Varian, Lexington, MA). Mobile phase A consisted of $0.1 \%$ formic acid, and mobile phase B consisted of $100 \%$ acetonitrile and $0.1 \%$ formic acid. The LTQ-Orbitrap mass spectrometer was operated in data-dependent acquisition mode using the Xcalibur 2.0.7 software. A single full-scan mass spectrum was conducted in the Orbitrap (400-1800 m/z, 30000 resolution), followed by 20 data-dependent MS/MS scans in the ion trap at $35 \%$ normalized collision energy (CID) or $3 \mathrm{MS} / \mathrm{MS}$ scans in the quadrupole collision cell with higher energy collision dissociation (HCD). MS/MS spectra from each LC-MS/MS run were searched against the selected database using an in-house Mascot or Proteome Discovery search algorithm.

\subsection{Calcineurin phosphatase assay}

All experiments were performed at $25^{\circ} \mathrm{C}$ in $1.8 \mathrm{~mL}$ reaction mixtures, and the time courses of absorbance changes were recorded using a Lambda 14 Perkin-Elmer spectrophotometer equipped with a magnetic stirrer in the cuvette holder. Initial rates were determined from the linear slope of the progress curves obtained, and the experimental data were analyzed using a nonlinear regression analysis program.

Phosphatase activity against p-RCAN1 and p-inhibitor-1 was detected by a continuous spectrophotometric assay. In this coupled system [22], the phosphate was transferred from p-RCAN1 or p-inhibitor-1 to 7-methyl-6-thioguanosine (MESG) by purine nucleoside phosphorylase. The reaction mixture contained $50 \mathrm{mmol} \mathrm{L}^{-1}$ MOPS pH 7.0, 100 mmol L ${ }^{-1} \mathrm{NaCl}, 0.1 \mathrm{mmol} \mathrm{L}^{-1}$ EDTA, $50 \mu \mathrm{mol} \mathrm{L}{ }^{-1} \mathrm{MESG}$, $0.1 \mathrm{mg} \mathrm{mL}^{-1}$ purine nucleoside phosphorylase, $1 \mathrm{mmol} \mathrm{L}^{-1}$ $\mathrm{CaCl}_{2}, 2 \mathrm{mmol} \mathrm{L}^{-1} \mathrm{MnCl}_{2}, 0.5 \mathrm{mg} \mathrm{mL}^{-1} \mathrm{BSA}, 1 \mathrm{mmol} \mathrm{L}{ }^{-1}$ DTT, and a range of concentrations of phospho-protein substrates, $10 \mu \mathrm{mol} \mathrm{L}{ }^{-1}$ of CaM was added to the p-RCAN1 assay and $1 \mu \mathrm{mol} \mathrm{L}{ }^{-1} \mathrm{CaM}$ was added to the p-inhibitor-1 assay. Dephosphorylation was detected by monitoring the absorbance change at $360 \mathrm{~nm}$ caused by the conversion of MESG to 7-methyl-6-thioguanine in the presence of inorganic phosphate. The quantitation of the phosphate release in the dephosphorylation reaction was thus determined using the extinction coefficient of $11200 \mathrm{M}^{-1} \mathrm{~cm}^{-1}$ at $360 \mathrm{~nm} \mathrm{[23].}$

\section{Results}

\subsection{RCAN1 noncompetitively inhibits calcineurin phosphatase activity}

RCANs represent a new class of endogenous calcineurin inhibitors [24,25]. RCAN1 may competitively inhibit the calcineurin-NFAT pathway in vivo because RCAN1 and
NFATs share an SP repeat motif and a PxIxIT motif. Recently, Chan et al. [26] demonstrated an inhibitory effect of human RCAN1 on calcineurin that competitively inhibited calcineurin activity against para-Nitrophenylphosphate (pNPP) substrate, a chromogenic compound. pNPP is a small-molecule substrate, and interference with this substrate strongly suggests a direct interaction between RCAN1 and the enzyme active site. To further examine whether the competitive inhibition is unique to small molecule aryl phosphates, we used a protein substrate, phospho-inhibitor1 , to evaluate the inhibitory properties of RCAN1 against calcineurin. Protein phosphatase inhibitor-1 is a prototypical mediator of cross talk between protein kinases and protein phosphatases. The activation of cAMP-dependent protein kinase results in the phosphorylation of inhibitor-1 at Thr-35, converting it to a protein substrate for calcineurin. For inhibition studies, the phospho-inhibitor-1 concentration was constant at $3.3 \mu \mathrm{mol} \mathrm{L}{ }^{-1}$, and the RCAN1 concentration was varied. The dephosphorylation of phospho-inhibitor-1 was measured using a continuous inorganic phosphate assay in which the $\mathrm{P}_{\mathrm{i}}$ produced by calcineurin is captured by purine ribonucleoside phosphorylase in the presence of a purine ribonucleoside substrate [22]. Figure 1 shows the effects of RCAN1 on the dephosphorylation of inhibitor-1 by calcineurin. A dose-response curve based on increasing concentrations of RCAN1 demonstrated that RCAN1 inhibited calcineurin activity (inset of Figure 1). However, the enzymatic activity decreased with an increase in the concentration of RCAN1 and approached a non-zero constant, indicating that binding of RCAN1 only partially inhibited calcineurin phosphatase activity against phospho-inhibitor-1. The apparent dissociation constant, $K_{\mathrm{i}}$, for the calcineurin inhibition reaction was $(0.42 \pm 0.01) \mu \mathrm{mol} \mathrm{L}^{-1}$.

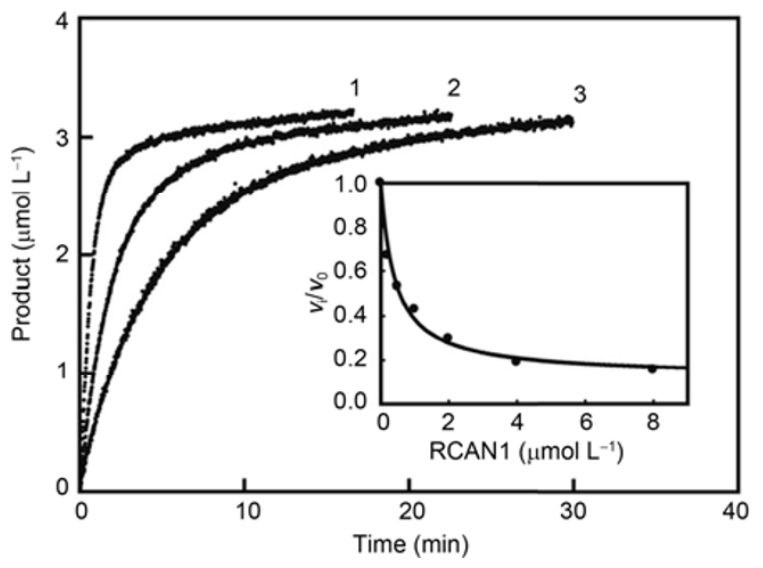

Figure 1 RCAN1 inhibits p-inhibitor-1 dephosphorylation by calcineurin in vitro. The figure shows the time courses of p-inhibitor-1 dephosphorylation by calcineurin with 0 (curve 1), 2 (curve 2) or $8 \mu \mathrm{mol} \mathrm{L}^{-1}$ (curve 3) RCAN1 and $3.3 \mu \mathrm{mol} \mathrm{L} \mathrm{L}^{-1}$ p-inhibitor-1. The calcineurin concentration used for the p-inhibitor- 1 assay was $0.192 \mu \mathrm{mol} \mathrm{L}^{-1}$. The inset shows the dependence of the inhibition of calcineurin-catalyzed p-inhibitor-1 dephosphorylation on the concentration of RCAN1. 


\subsection{RCAN1 is phosphorylated by p38a MAP kinase at multiple residues}

A previous study identified two $\mathrm{S}$ residues in human RCAN1, S108 and S112, as substrates of glycogen synthase kinase $3 \beta$ (GSK-3 $\beta$ ) and ERK2 MAP kinase, respectively [27]. These residues lie within the highly conserved FLISPPxSPP signature motif of the RCAN family. To investigate whether $\mathrm{p} 38 \alpha$ MAP kinase could also phosphorylate RCAN1, in vitro kinase reactions were performed with either WT or mutant (S108A, S112A or S108A/S112A) recombinant RCAN1 proteins and recombinant activated p38 $\alpha$ MAP kinase in a kinase assay buffer containing ATP and $\mathrm{Mg}^{2+}$, and the electrophoretic mobility of RCAN1 was determined by SDS-PAGE (Figure 2). At the appropriate time points, aliquots of the reaction mixture were withdrawn, and the reaction was terminated with an equal volume of $2 \times$ Laemmli SDS sample buffer, followed by separation on a 15\% SDS-PAGE gel. As shown in Figure 2B, RCAN1 exhibited a time-dependent change in phosphorylation, as indicated by the electrophoretic mobility shift. The p38 $\alpha$ MAP kinase-catalyzed phosphorylation was nearly $100 \%$ complete at $30 \mathrm{~min}$, and the fully phosphorylated RCAN1 protein ran as a single band on a $15 \%$ SDS-PAGE gel.

To identify specific RCAN1 residues that were phosphorylated by $\mathrm{p} 38 \alpha$ MAP kinase, we purified the p38 $\alpha$ MAP kinase-phosphorylated RCAN1 WT and RCAN1 S108A/S112A proteins and performed electrospray mass spectrometry. As shown in Figure 3 and Figure 4, electrospray spectrometry of a tryptic digestion of phosphoRCAN1 revealed five phosphorylation sites in WT RCAN1 (S93, S108, S112, T124 and T153) (Figure 3) and three sites in RCAN1 S108A/S112A (S93, T124 and T153) (Figure 4).

\section{3 p38a MAP kinase-phosphorylated RCAN1 is a good substrate for calcineurin}

We next determined whether p38 $\alpha$ MAP kinase-phosphorylated RCAN1 could serve as a calcineurin substrate using a continuous spectrophotometric assay. In this method, the phosphorylation level of RCAN1 was measured by the amount of inorganic phosphate released from RCAN1 after treatment with calcineurin. The inorganic phosphate produced was monitored continuously by a coupled enzyme assay using purine nucleoside phosphorylase and its chromophoric substrate, MESG, and quantitated using the extinction coefficient of $11200 \mathrm{M}^{-1} \mathrm{~cm}^{-1}$ at $360 \mathrm{~nm}$. Figure 5A shows time courses for the dephosphorylation of phospho-RCAN1 by calcineurin. The data in this figure show that the calcineurin-catalyzed reaction was nearly $100 \%$ complete in $15 \mathrm{~min}$. When all of the substrate had been converted to product, the phosphorylation stoichiometry of
RCAN1 was determined to be close to $5 \mathrm{~mol}$ phosphate $/ \mathrm{mol}$ RCAN1. As expected, only 3 equivalents of inorganic phosphate were released upon treatment of the S108A/ S112A mutant with calcineurin. These results further support our conclusion that p38 $\alpha$ MAP kinase can phosphorylate RCAN1 at multiple residues in vitro.

To further characterize the biochemical properties of phospho-RCAN1, we measured the kinetic parameters of the calcineurin-catalyzed reaction using phosphorylated RCAN1 and the S108A/S112A mutant as substrates. All steady-state kinetic studies were performed at $\mathrm{pH} 7.0$ and $25^{\circ} \mathrm{C}$. The initial rates of the dephosphorylation reaction were determined from the slopes of the initial linear portion of the progress curves. Figure 5B shows the initial rates of the calcineurin-catalyzed reaction at different RCAN1 concentrations. Because the concentrations of RCAN1 and calcineurin were comparable in the reaction, the assumption that the free substrate concentration was equal to the total substrate concentration could not be made. Thus, the kinetic data were analyzed using the general initial velocity equation, taking substrate depletion into account.

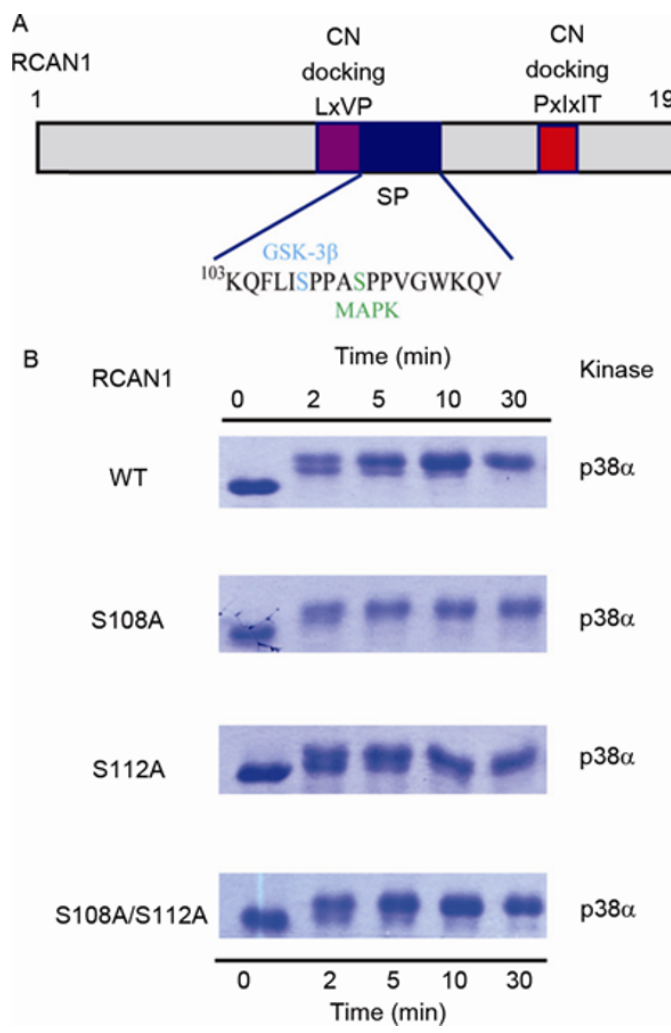

Figure 2 Phosphorylation of RCAN1 by p38 $\alpha$ MAP kinase. A, Phosphorylation sites for MAPK and GSK-3 $\beta$ are present in the SP repeat of RCAN1. The serine phosphorylated by MAPK is shown in green, while the GSK- $3 \beta$ phosphorylation site is shown in cyan. The LxVP docking site, SP motif and PxIxIT docking sites are shown in purple, blue and red, respectively. B, Time courses of RCAN1 WT and mutant phosphorylation by p38 $\alpha$ MAP kinase. RCAN1 was phosphorylated in an in vitro phosphorylation system (see "Materials and methods") containing $40 \mu \mathrm{mol} \mathrm{L}{ }^{-1}$ RCAN1 and $2 \mu \mathrm{mol} \mathrm{L}{ }^{-1}$ p38 $\alpha$ MAP kinase. 

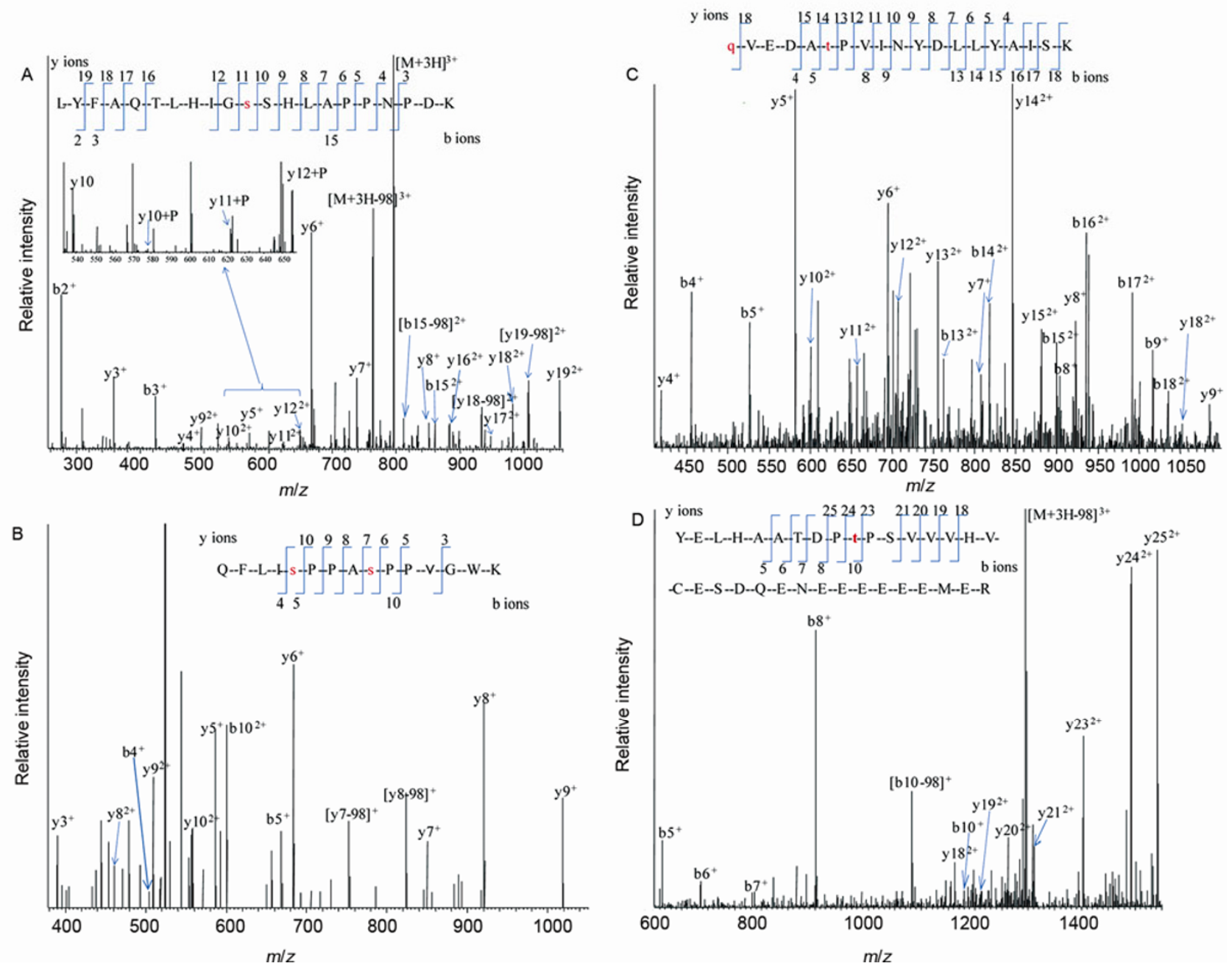

Figure 3 Identification of $\mathrm{p} 38 \alpha$ MAP kinase phosphorylation sites of RCAN1 by mass spectrometry. A, The MS/MS spectrum of a triply charged ion at $\mathrm{m} / z 796.39$ for $\mathrm{MH}_{3}{ }^{3+}$ corresponding to the mass of the mono-phosphorylated peptide LYFAQTLHIGSSHLAPPNPDK. A loss of $98 \mathrm{Da}\left(\mathrm{H}_{3} \mathrm{PO}_{4}\right)$ was observed for the precursor ion under the higher energy collision dissociation (HCD). The labeled peaks correspond to the masses of the $y$ and $b$ ions of the modified peptides. The y10 ion was observed to have high intensity compared to the phosphorylated y10 ion, as shown in the inserted figure, suggesting that Ser-11 is the major phosphorylated residue. B. The MS/MS spectrum of a triply charged ion at $\mathrm{m} / \mathrm{z} 595.61$ for $\mathrm{MH}_{3}{ }^{3+}$ corresponding to the mass of the di-phosphorylated peptide QFLISPPASPPVGWK. The labeled peaks correspond to the masses of the $\mathrm{b}$ and $\mathrm{y}$ ions of the di-phosphorylated peptide, indicating that both Ser residues are phosphorylated. C, The MS/MS spectrum of a triply charged ion at $\mathrm{m} / \mathrm{z} 739.36$ for $\mathrm{MH}_{3}{ }^{3+}$ corresponding to the mass of the mono-phosphorylated peptide qVEDATPVINYDLLYAISK (in which the first residue Gln is a pyroglutamine). The labeled peaks correspond to the masses of the $\mathrm{b}$ and $\mathrm{y}$ ions of the mono-phosphorylated peptide, indicating that phosphorylation is on the Thr-6 residue. D, The MS/MS spectrum of a triply charged ion at $m / z 1328.55$ for $\mathrm{MH}_{3}{ }^{3+}$, corresponding to the mass of a mono-phosphorylated peptide YELHAATDPTPSVVVHVCESDQENEE- EEEEMER in which

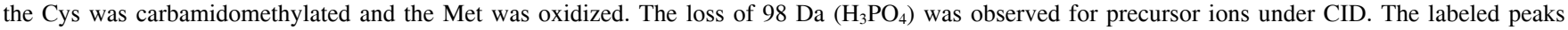
correspond to the masses of the $\mathrm{b}$ and $\mathrm{y}$ ions of the mono-phosphorylated peptide, indicating that phosphorylation is on the Thr-10 residue.

$v_{0}=\frac{k_{\text {cat }}}{2}\left\{[S]_{0}+[E]_{0}+K_{\mathrm{m}}-\sqrt{\left\{[E]_{0}+[S]_{0}+K_{\mathrm{m}}\right\}^{2}-4[E]_{0}[S]_{0}}\right\}$.

Direct curve fitting of the data to eq. (1) yielded the $k_{\text {cat }}$ and $K_{\mathrm{m}}$ values for the dephosphorylation of RCAN1 and the S108A/S112A mutant by calcineurin (Table 1 ). The $K_{\mathrm{m}}$ values are approximately 7 -fold greater than the inhibition constant $K_{\mathrm{i}}$, indicating that unphosphorylated RCAN1 appeared to bind more strongly to calcineurin than $\mathrm{p} 38 \alpha \mathrm{MAP}$ kinase-phosphorylated RCAN1. The substrate specificity constant $k_{\text {cat }} / K_{\mathrm{m}}$ of RCAN1 was calculated as $(0.59 \pm 0.15) \times$
Table 1 Kinetic parameters of calcineurin toward different substrates

\begin{tabular}{ccc}
\hline Substrate & $K_{\mathrm{m}}\left(\mu \mathrm{mol} \mathrm{L}{ }^{-1}\right)$ & $k_{\text {cat }}\left(\mathrm{s}^{-1}\right)$ \\
\hline RCAN1 WT & $2.697 \pm 0.516$ & $0.158 \pm 0.009$ \\
RCAN1 S108AS112A & $4.456 \pm 0.313$ & $0.148 \pm 0.004$ \\
NFAT $^{\mathrm{a})}$ & 2.04 & 0.11 \\
\hline
\end{tabular}

a) NFAT data are from a published paper [28].

$10^{5} \mathrm{M}^{-1} \mathrm{~s}^{-1}$, which was similar to that of the calcineurincatalyzed NFAT dephosphorylation [28], suggesting that p38 $\alpha$ MAP kinase-phosphorylated RCAN1 may be a physiological substrate for calcineurin in vivo. 


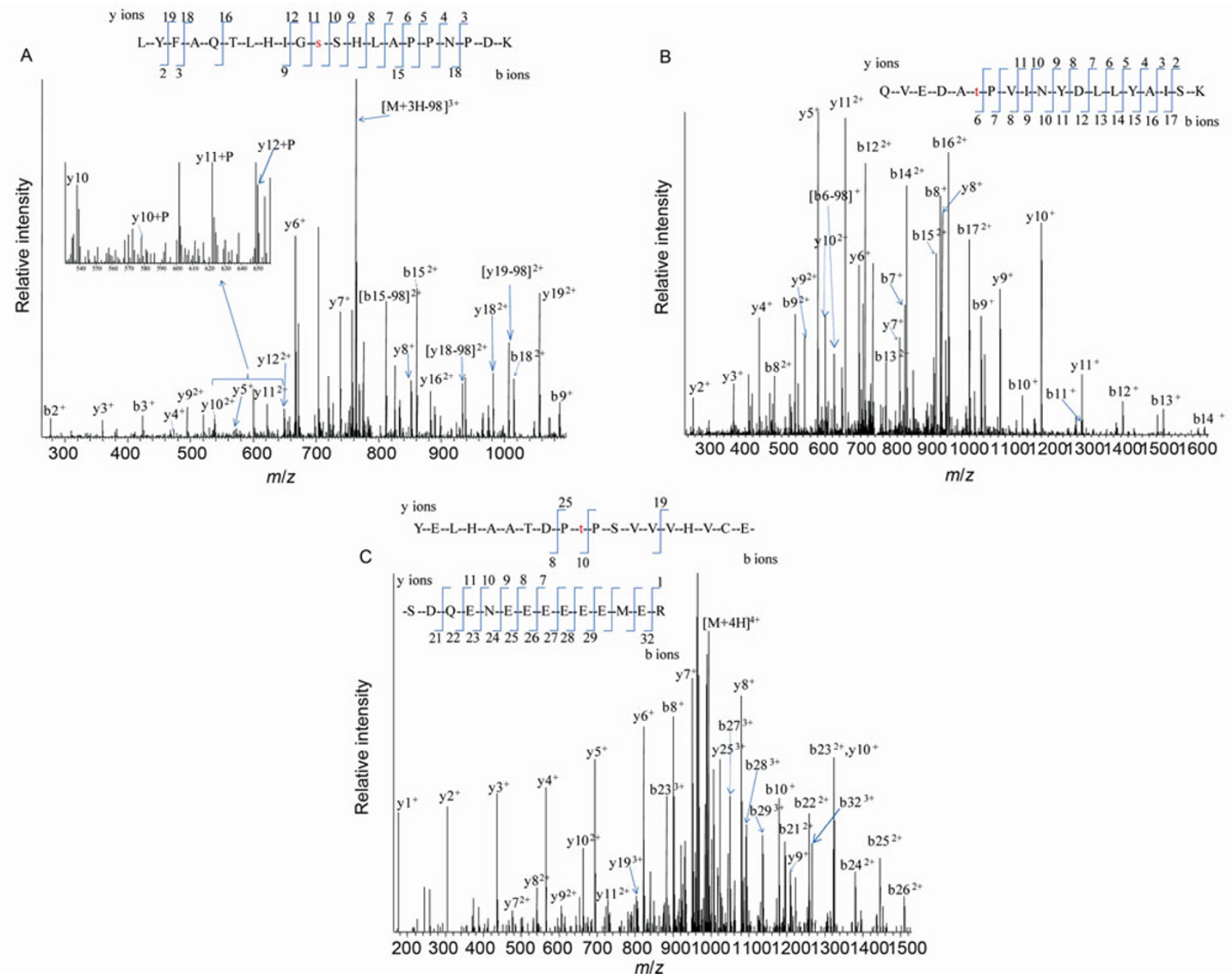

Figure 4 Identification of $\mathrm{p} 38 \alpha$ MAP kinase phosphorylation sites in RCAN1 S108A/S112A by mass spectrometry. A, The MS/MS spectrum of a triply charged ion at $\mathrm{m} / \mathrm{z} 796.39$ for $\mathrm{MH}_{3}{ }^{3+}$ corresponding to the mass of the mono-phosphorylated peptide LYFAQTLHIGSSHLAPPNPDK. A loss of 98 Da $\left(\mathrm{H}_{3} \mathrm{PO}_{4}\right)$ was observed for the precursor ion under the higher energy collision dissociation (HCD). The labeled peaks correspond to the masses of the $\mathrm{y}$ and $\mathrm{b}$ ions of the modified peptides. The y10 ion was observed to have a high intensity compared with the phosphorylated y10 ion, as shown in the inserted figure, suggesting that Ser-11 is the major phosphorylated residue. B, The MS/MS spectrum of a triply charged ion at $m / z, 745.03$ for $\mathrm{MH}_{3}{ }^{3+}$ corresponding to the mass of the mono-phosphorylated peptide QVEDATPVINYDLLYAISK. The labeled peaks correspond to the masses of the $b$ and $y$ ions of the mono-phosphorylated peptide, indicating that phosphorylation is on the Thr-6 residue. C, The MS/MS spectrum of a quadruply charged ion at $m / z$ 993.18 for $\mathrm{MH}_{3}{ }^{3+}$ corresponding to the mass of the mono-phosphorylated peptide YELHAATDPTPSVVVHVCESD- QENEEEEEEMER in which the Cys residue was carbamidomethylated. The loss of $98 \mathrm{Da}\left(\mathrm{H}_{3} \mathrm{PO}_{4}\right)$ was observed for precursor ions under $\mathrm{HCD}$. The labeled peaks correspond to the masses of the $\mathrm{b}$ and $\mathrm{y}$ ions of the mono-phosphorylated peptide, indicating that phosphorylation is on the Thr-10 residue.

\section{Discussion}

RCANs were first identified using screens for calcineurin binding proteins in fungi [25]. The overexpression of the yeast protein RCN1 or human RCAN1 inhibited at least two independent functions of calcineurin in yeast, the activation of the transcription factor Tcnlp and the inhibition of the $\mathrm{H}^{+} / \mathrm{Ca}^{2+}$ exchanger Vcxlp. Signaling via calmodulin, calcineurin, and Tcn1p induced RCN1 expression, suggesting that RCN1 operates as an endogenous feedback inhibitor of calcineurin [24]. Human RCAN1 proteins are expressed in diverse cell types, including neurons and muscles [29-31], and have complex effects in cells. However, the precise mechanisms and detailed roles of RCANs in calcineurin signaling are still unclear.

RCAN1 has been reported to be phosphorylated by various kinases, such as glycogen synthase kinase 3 (GSK-3) [27,32], ERK2 MAP kinase [27], big MAP kinase 1 (BMK1) [33], NFkB-inducing kinase (NIK) [34], TGF- $\beta$-activated kinase 1 (TAK1) [35], dual specificity tyrosine phosphorylation-regulated kinase 1A (Dyrk1A) [36] and protein kinase A (PKA) [37]. The phosphorylation of RCAN1 has been shown to regulate calcineurin activity both positively and negatively. The central region of RCAN1 contains a perfect match to the GSK-3 consensus phosphorylation site (S108) [27] in the SP repeat of RCAN1. Phosphorylation by 

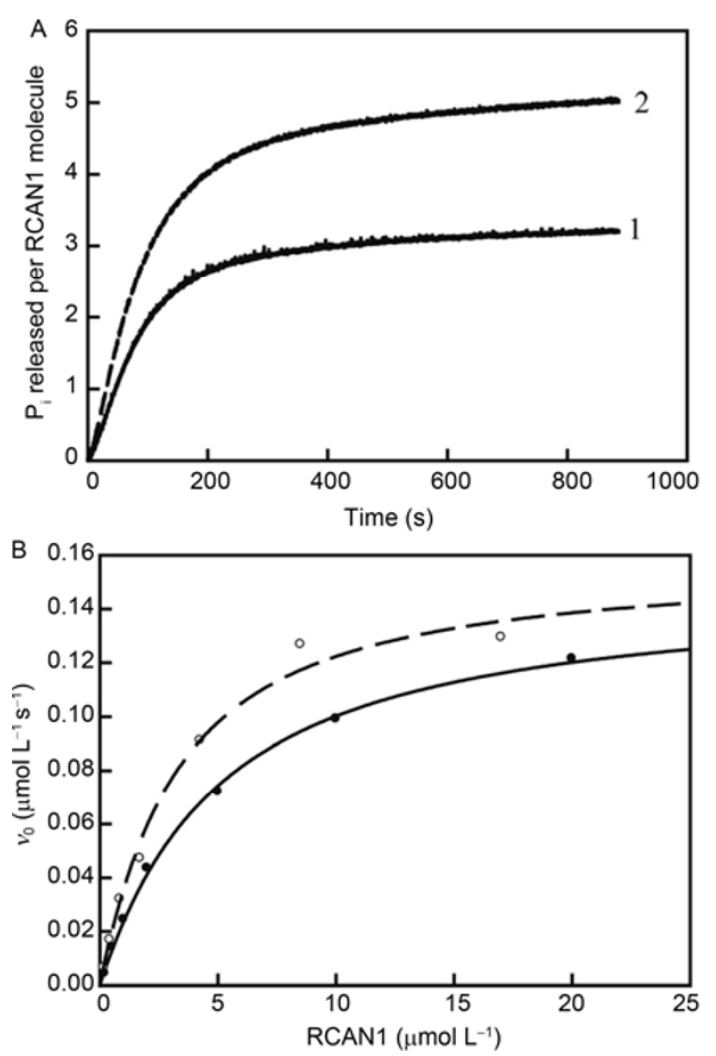

Figure 5 Dephosphorylation of RCAN1 WT and S108A/S112A by calcineurin. A, The time courses of p-RCAN1 WT (curve 2) and S108A/ S112A (curve 1) dephosphorylation by calcineurin. B, The dependence of the initial rate of the calcineurin-catalyzed reaction on the concentration of p-RCAN1 WT (open circle) and p-RCAN1 S108A/S112A (solid circle). 1 $\mu \mathrm{mol} \mathrm{L}{ }^{-1}$ calcineurin was used as the phosphatase.

GSK-3 requires a priming phosphorylation at a nearby site (S112) by ERK2 [27]. The GSK-3-mediated phosphorylation of RCAN1 activates calcineurin signaling in vivo [32]. Similarly, TAK1 phosphorylates RCAN1 at two positions, S94 and S136, converting it from an inhibitor to a facilitator of calcineurin-NFAT signaling [35]. BMK1 can phosphorylate RCAN1 at S112, and the BMK1-mediated phosphorylation of RCAN1 promotes the dissociation of RCAN1 from calcineurin, thereby relieving its inhibition of calcineurin activity [33]. In contrast, a recent report showed that Dyrk1A can phosphorylate RCAN1 at S112 and T192, and the phosphorylation of RCAN1 at T192 by Dyrk1A increases its ability to inhibit calcineurin phosphatase activity [36]. The PKA-mediated phosphorylation of RCAN1 at an unknown phosphorylation site has also been shown to enhance its inhibition of calcineurin-mediated gene transcription [37]. In addition to regulating calcineurin activity, the phosphorylation of RCAN1 affects its protein stability through the ubiquitin-proteasome pathway [38]. The phosphorylation of RCAN1 at the FLISPP motif controls the half-life of RCAN1 by accelerating its degradation, while phosphorylation of the C-terminal region by NIK blocks degradation and increases the stability of the protein.
In the present study, we examined the putative role of $\mathrm{p} 38 \alpha$ MAP kinase in the regulation of RCAN1 activity. We found that $\mathrm{p} 38 \alpha$ MAP kinase phosphorylates RCAN1 on multiple sites both within and outside the FLISPP motif in vitro (S93, S108, S112, T124 and T153). Thus, p38 $\alpha$ MAP kinase-mediated RCAN1 phosphorylation may represent a previously unrecognized MAP kinase cascade that regulates calcineurin signaling. The physiological significance of the MAP kinase regulation of calcineurin activation through the phosphorylation of RCAN1 will be an interesting topic for future studies.

This work was supported in part by Ministry of Science and Technology of China (Grant 2011CB910803).

1 Musson R E, Smit N P. Regulatory mechanisms of calcineurin phosphatase activity. Curr Med Chem, 2011, 18: 301-315

2 Shi Y. Serine/threonine phosphatases: Mechanism through structure. Cell, 2009, 139: 468-484

3 Reese L C, Taglialatela G. A role for calcineurin in Alzheimer's disease. Curr Neuropharmacol, 2011, 9: 685-692

4 Clipstone N A, Crabtree G R. Identification of calcineurin as a key signalling enzyme in T-lymphocyte activation. Nature, 1992, 357: 695-697

5 Wang H G, Pathan N, Ethell I M, et al. $\mathrm{Ca}^{2+}$-induced apoptosis through calcineurin dephosphorylation of bad. Science, 1999, 284: 339-343

6 Dunn S E, Burns J L, Michel R N. Calcineurin is required for skeletal muscle hypertrophy. J Biol Chem, 1999, 274: 21908-21912

7 Bear M F, Malenka R C. Synaptic plasticity: Ltp and ltd. Curr Opin Neurobiol, 1994, 4: 389-399

8 Mansuy I M, Mayford M, Jacob B, et al. Restricted and regulated overexpression reveals calcineurin as a key component in the transition from short-term to long-term memory. Cell, 1998, 92: 39-49

9 Molkentin J D, Lu J R, Antos C L, et al. A calcineurin-dependent transcriptional pathway for cardiac hypertrophy. Cell, 1998, 93: 215228

10 Loh C, Shaw K T, Carew J, et al. Calcineurin binds the transcription factor nfat1 and reversibly regulates its activity. J Biol Chem, 1996, 271: 10884-10891

11 Masuda E S, Liu J, Imamura R, et al. Control of nfatx1 nuclear translocation by a calcineurin-regulated inhibitory domain. Mol Cell Biol, 1997, 17: 2066-2075

12 Liu J, Masuda E S, Tsuruta L, et al. Two independent calcineurin-binding regions in the n-terminal domain of murine nf-atx 1 recruit calcineurin to murine nf-atx1. J Immunol, 1999, 162: 47554761

13 Martinez-Martinez S, Rodriguez A, Lopez-Maderuelo M D, et al. Blockade of NFAT activation by the second calcineurin binding site. J Biol Chem, 2006, 281: 6227-6235

14 Macian F, Lopez-Rodriguez C, Rao A. Partners in transcription: NFAT and AP-1. Oncogene, 2001, 20: 2476-2489

15 Chang K T, Shi Y J, Min K T. The drosophila homolog of down's syndrome critical region 1 gene regulates learning: Implications for mental retardation. Proc Natl Acad Sci USA, 2003, 100: 1579415799

16 Harris C D, Ermak G, Davies K J. Rcan1-11 is overexpressed in neurons of Alzheimer's disease patients. FEBS J, 2007, 274: 17151724

17 Baek K H, Zaslavsky A, Lynch R C, et al. Down's syndrome suppression of tumour growth and the role of the calcineurin inhibitor DSCR1. Nature, 2009, 459: 1126-1130

18 Arron J R, Winslow M M, Polleri A, et al. NFAT dysregulation by increased dosage of DSCR1 and DYRK1A on chromosome 21. Na- 
ture, 2006, 441: 595-600

19 Ryeom S, Baek K H, Rioth M J, et al. Targeted deletion of the calcineurin inhibitor DSCR1 suppresses tumor growth. Cancer Cell, 2008, 13: 420-431

20 Killilea S D, Cheng Q, Wang Z X. Protein phosphatase type 1 and type 2a assays. Methods Mol Biol, 1998, 93: 23-33

21 Zhang Y Y, Wu J W, Wang Z X. Mitogen-activated protein kinase (MAPK) phosphatase 3-mediated cross-talk between MAPKs ERK2 and p38alpha. J Biol Chem, 2011, 286: 16150-16162

22 Webb M R. A continuous spectrophotometric assay for inorganic phosphate and for measuring phosphate release kinetics in biological systems. Proc Natl Acad Sci USA, 1992, 89: 4884-4887

23 Sergienko E A, Srivastava D K. A continuous spectrophotometric method for the determination of glycogen phosphorylase-catalyzed reaction in the direction of glycogen synthesis. Anal Biochem, 1994, 221: $348-355$

24 Kingsbury T J, Cunningham K W. A conserved family of calcineurin regulators. Genes Dev, 2000, 14: 1595-1604

25 Gorlach J, Fox D S, Cutler N S, et al. Identification and characterization of a highly conserved calcineurin binding protein, CBP1/calcipressin, in cryptococcus neoformans. EMBO J, 2000, 19: 3618-3629

26 Chan B, Greenan G, McKeon F, et al. Identification of a peptide fragment of DSCR1 that competitively inhibits calcineurin activity in vitro and in vivo. Proc Natl Acad Sci USA, 2005, 102: 13075-13080

27 Vega R B, Yang J, Rothermel B A, et al. Multiple domains of MCIP1 contribute to inhibition of calcineurin activity. J Biol Chem, 2002, 277: 30401-30407

28 Kilka S, Erdmann F, Migdoll A, et al. The proline-rich N-terminal sequence of calcineurin abeta determines substrate binding. Biochemistry, 2009, 48: 1900-1910

29 Ermak G, Morgan T E, Davies K J. Chronic overexpression of the calcineurin inhibitory gene DSCR1 (Adapt78) is associated with Alzheimer's disease. J Biol Chem, 2001, 276: 38787-38794

30 Casas C, Martinez S, Pritchard M A, et al. DSCR1, a novel endogenous inhibitor of calcineurin signaling, is expressed in the primitive ventricle of the heart and during neurogenesis. Mech Dev, 2001, 101: 289-292

31 Rothermel B, Vega R B, Yang J, et al. A protein encoded within the down syndrome critical region is enriched in striated muscles and inhibits calcineurin signaling. J Biol Chem, 2000, 275: 725

32 Hilioti Z, Gallagher D A, Low-Nam S T, et al. GSK-3 kinases enhance calcineurin signaling by phosphorylation of RCNS. Genes Dev, 2004, 18: 35-47

33 Abbasi S, Lee J D, Su B, et al. Protein kinase-mediated regulation of calcineurin through the phosphorylation of modulatory calcineurin-interacting protein 1. J Biol Chem, 2006, 281: 7717-7726

34 Lee E J, Seo S R, Um J W, et al. NF-kappaB-inducing kinase phosphorylates and blocks the degradation of down syndrome candidate region 1. J Biol Chem, 2008, 283: 3392-3400

35 Liu Q, Busby J C, Molkentin J D. Interaction between TAK1-TAB1TAB2 and RCAN1-calcineurin defines a signalling nodal control point. Nat Cell Biol, 2009, 11: 154-161

36 Jung M S, Park J H, Ryu Y S, et al. Regulation of RCAN1 protein activity by DYRK1a protein-mediated phosphorylation. J Biol Chem, 2011, 286: 40401-40412

37 Kim S S, Oh Y, Chung K C, et al. Protein kinase a phosphorylates down syndrome critical region 1 (RCAN1). Biochem Biophys Res Commun, 2012, 418: 657-661

38 Kishi T, Ikeda A, Nagao R, et al. The SCFCDC4 ubiquitin ligase regulates calcineurin signaling through degradation of phosphorylated RCN1, an inhibitor of calcineurin. Proc Natl Acad Sci USA, 2007, 104: 17418-17423

Open Access This article is distributed under the terms of the Creative Commons Attribution License which permits any use, distribution, and reproduction in any medium, provided the original author(s) and source are credited. 